\section{The COVID-19 pandemic and traffic accidents}

To the Editor: We read the article entitled 'The effect of lockdown on intentional and non-intentional injury during the COVID-19 pandemic in Cape Town, South Africa: A preliminary report ${ }^{[1]}$ with a great interest. Navsaria et al. ${ }^{[1]}$ concluded that 'there was an overall trend of reduced number of patients who visited the trauma unit during the hard lockdown period; however, these numbers returned to pre-lockdown levels during the immediate post-lockdown period. The effect of COVID-19 and COVID-19 lockdown on accident epidemiology is an interesting issue. A lockdown would be expected to result in less traffic on the road, which should be associated with reduced numbers of accidents and lower accident-related mortality. We would like to share observations from Thailand, a country in Indochina. Lockdown was implemented there during April - October 2020. Then there was a month without lockdown (November 2020) before the second wave of COVID-19 and implementation of strict control measures from December 2020. We present data for three long (5-day) public holiday periods: preCOVID-19 (New Year 2020), during the first COVID-19 lockdown (the local cultural New Year in April 2020), and during the second COVID-19 lockdown (New Year 2021). The mortality rates due to accidents during these three holiday periods were 63.7, 33.4 and 71.6 per day, respectively. There was a significant reduction in accidentrelated mortality during the first COVID-19 lockdown period, but no reduction during the second period of strict control - in fact, there were more deaths than in the pre-COVID period. These figures may indicate that people limited their movement due to a state of panic during the first lockdown, but that they had come to accept the outbreak situation and to ignore transport restrictions by the second lockdown. The mortality rate was expected to be lower during the first lockdown, but restrictions may have resulted in difficulties in rescue activity, which would affect mortality among people injured in road traffic accidents. ${ }^{[2]}$

\section{Rujittika Mungmunpuntipantip}

Private academic consultant, Bangkok, Thailand rujittika@gmail.com

\section{Viroj Wiwanitkit}

Honorary Professor, Dr D Y Patil University, Pune, India; and Adjunct Professor, Joseph Ayobabalola University, Ikeji-Arakeji, Nigeria

1. Navsaria PH, Nicol AJ, Parry CDH, Matzopoulos R, Maqungo S, Gaudin R. The effect of lockdown on intentional and non-intentional injury during the COVID-19 pandemic in Cape Town, South Africa: A preliminary report. S Afr Med J 2021;111(2):110-113. https://doi.org/10.7196/SAMJ.2021. v111i2.15318

2. Dicker B, Swain A, Todd VF, et al. Changes in demand for emergency ambulances during a nationwide lockdown that resulted in elimination of COVID-19: An observational study from New Zealand. BMJ Open 2020;10(12):e044726. https://doi.org/10.1136/bmjopen-2020-044726

S Afr Med J 2021;111(3):192. https://doi.org/10.7196/SAMJ.2021.v111i3.15531 